\title{
Enquête
}

Archives de la revue Enquête

7| 1999

Les objets du droit

\section{Le génome entre nature des choses et artefact}

The Genome between the Nature of Things and the Artefact

\section{Florence Bellivier}

\section{OpenEdition}

\section{Journals}

Édition électronique

URL : http://journals.openedition.org/enquete/1566

DOI : 10.4000/enquete. 1566

ISSN : 1953-809X

\section{Éditeur :}

Cercom, Éditions Parenthèses

\section{Édition imprimée}

Date de publication : 1 novembre 1999

Pagination : 55-72

\section{Référence électronique}

Florence Bellivier, « Le génome entre nature des choses et artefact », Enquête [En ligne], 7| 1999, mis en ligne le 15 juillet 2013, consulté le 19 avril 2019. URL : http://journals.openedition.org/ enquete/1566 ; DOI : 10.4000/enquete.1566

Ce document a été généré automatiquement le 19 avril 2019 


\title{
Le génome entre nature des choses et artefact
}

\author{
The Genome between the Nature of Things and the Artefact
}

Florence Bellivier

1 Le génome humain est constitué par l'ensemble des gènes, autrement dit des entités qui, considérées d'un point de vue matériel et informatif, sont responsables d'un caractère repérable dans un organisme vivant. Chaque gène est une portion d'ADN (acide désoxyribonucléique), c'est-à-dire du support moléculaire de l'hérédité, dont la capacité de duplication assure la transmission, d'une génération à l'autre, des caractères de tel ou tel organisme. Dès lors, il est essentiel de comprendre que le génome humain doit être envisagé de deux points de vue : individuel (le génome de cet individu-ci, résultante de celui de chacun de ses deux parents biologiques) et collectif (le génome de telle famille, de telle population, voire de l'espèce entière).

2 Ainsi brièvement défini, il présente deux intérêts majeurs pour qui s'attache à la construction de l'objet en droit. Tout d'abord, il nous permet d'assister à une construction juridique «en direct», même si elle est parfois encore elliptique: ce n'est que très récemment, d'abord par le biais de certaines décisions de justice puis au travers des lois du 29 juillet 1994 sur le statut du corps humain et la réglementation des activités portant sur ce dernier, ainsi que de textes internationaux, que le génome a été pris en compte par le droit. En effet, il est désormais l'objet de pratiques telles que le séquençage, la thérapie génique, les différents diagnostics précédant la naissance, notamment le diagnostic préimplantatoire, ou encore les procréations médicalement assistées ; or d'une façon ou d'une autre, chacune de ces techniques implique la mise à disposition du génome et, par là même, rend nécessaire un encadrement. De ce corpus - en voie de constitution - de textes et de décisions se dégagent diverses qualifications, explicites ou implicites, de l'objet « génome ».

3 Le second intérêt du génome quant au processus de la construction de l'objet en droit, réside dans la spécificité de l'entité en question, à la frontière de plusieurs dimensions 
difficiles à articuler entre elles : non seulement, on l'a vu, le collectif et l'individuel, mais aussi le naturel et l'artificiel, l'abstrait et le concret. En ce qui concerne cette dernière opposition, on ne saurait entamer une réflexion sur le génome sans envisager, ne seraitce que brièvement, son appréhension par les spécialistes au premier chef intéressés, à savoir les biologistes. Manipulé par ces derniers, le génome est, bien entendu, tout d'abord une réalité matérielle, contenue dans tel tissu, telle cellule, etc. Toutefois, cette réalité ne leur parvient qu'au moyen de procédés très sophistiqués qui, d'une certaine manière, contribuent à rendre le génome abstrait. Il n'est que de penser au séquençage du génome humain, combinaison de techniques relevant de la biologie moléculaire et de l'informatique, par lesquelles les gènes sont déroulés et décodés d'une façon qui en manifeste toute l'abstraction. À cet égard, on soulignera que, pour un biologiste, il n'y a pas de différence fondamentale entre le génome de l'individu et celui de l'espèce, ni même entre le génome humain et le génome animal ou végétal.

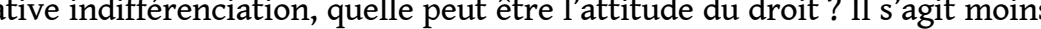
d'évoquer ici la réglementation juridique du génome que de se demander comment la vision qu'a le juriste de ce dernier vient s'articuler sur celle du biologiste, pour y adhérer ou, au contraire, la déformer. Le juriste entérine-t-il les conceptions biologiques du génome ? Les reconstruit-il à sa façon ? C'est à ce stade, plus qu'à celui de l'étude générale de la réglementation du patrimoine génétique, que l'on peut tenter d'appréhender la construction juridique de cet objet. À cet égard, il paraît intéressant de s'attacher à l'une des rares qualifications explicites qui en aient été données, celle de patrimoine commun de l'humanité. Bien qu'il figure dans un texte de faible valeur contraignante, la Déclaration universelle sur le génome humain et les droits de l'homme, adoptée par l'Unesco en 1997, ce parti pris est digne d'être commenté car en lui se rassemblent nombre de difficultés et de contradictions suscitées par la qualification juridique de l'objet " génome ». En énonçant que «Le génome humain sous-tend l'unité fondamentale de tous les membres de la famille humaine, ainsi que la reconnaissance de leur dignité et de leur diversité » et que "Dans un sens symbolique, il est le patrimoine commun de l'humanité ${ }^{1}$, l'Unesco se situe dans une double perspective très claire. D'une part, d'un point de vue moral et politique, les rédacteurs du texte ont eu à l'esprit les dangers de discrimination (sociale, raciale, etc.) que les pratiques ayant le génome pour objet, sinon portent en germe, du moins peuvent provoquer, par exemple en matière d'assurance ou d'emploi. Loin de vouloir prohiber la recherche sur le génome, la Déclaration s'est alors avant tout voulu œuvre d'équilibre entre ce qui est faisable techniquement et ce qui est permis (par la morale commune, relayée par la loi). D’autre part, l'Unesco prévient les objections généralement formulées à l'encontre de la qualification « patrimoine commun de l'humanité », en précisant qu'elle en fait ici un usage "symbolique ». L'expression n'est donc pas à prendre au pied de la lettre, elle est surtout révélatrice d'une intention : autant un symbole, en tant que signe représentant un concept, peut être riche, autant le simple usage "symbolique » d'une notion manifeste un appauvrissement de sens. Qu'on se le tienne pour dit: le génome ne sera pas approprié par quiconque, pas même par l'humanité, cette abstraction qui masque mal le déséquilibre réel des rapports entre les divers pays membres de l'Unesco.

5 Pour louable qu'elle soit, cette qualification suscite d'irritantes difficultés, et ce quel que soit le registre où l'on entend la situer (moral, symbolique, juridique). D'un côté, l'on est tenté de qualifier le génome par référence à la nature, point d'appui assez vacillant mais signifiant, au minimum, deux choses : le génome est inné, il est le résultat de la grande 
loterie de la nature - par quoi l'on retrouve l'étymologie du mot «nature »-; manifestation biologique de la singularité de chacun, il permet aussi d'inscrire tout individu dans une communauté plus vaste, notamment celle de l'espèce, concept biologique mettant en relief l'inclusion de l'individu dans un ordre reproductif qui le dépasse. On appellera "naturalisante» cette conception du génome fondée sur une référence à la nature comme donné primordial (l'hérédité reçue, bonne ou mauvaise) et comme horizon d'action (l'espèce, dont la vocation est de se reproduire et dont on essaie d'assurer l'intégrité). De l'autre côté, il est difficile d'ignorer la nature d'artefact du génome $e^{2}$ en tant que produit de l'action technique de l'homme, plus précisément en tant qu'objet naturel modifié par ce dernier. Invisibles à l'œil nu, intangibles - au contraire du corps -, mis au jour par les biologistes au moyen d'outils très perfectionnés, les gènes, qui à leur tour servent à fabriquer des substances (pensons à l'insuline humaine recombinante issue de bactéries et destinée aux diabétiques), sont bien devenus des productions techniques, elles-mêmes issues de savoirs abstraits. Si l'on songe alors que le droit est fondamentalement reconstruction du réel à travers ses concepts, ses catégories, voire ses fictions, on peut alors fort bien imaginer pour le génome des qualifications qui, loin d'épouser sa naturalité, en redoubleraient l'artificialité. Mais, tel n'est pas le sens de la qualification étudiée ici, celle de l'Unesco, qui, pour critiquable qu'elle soit, n'en manifeste pas moins de façon éclatante à la fois l'irréductible «naturalité » du génome, reflet d'une irréductibilité plus générale de la nature en droit, et les insuffisances, pour la construction juridique du génome, des qualifications « naturalisantes » de ce dernier.

\section{L'irréductible « naturalité » du génome}

6 Il faut commencer par expliquer en quoi la qualification, fût-elle «symbolique », du génome comme « patrimoine commun de l'humanité » révèle l'ambiguïté du droit dans sa référence à la nature en ce qui concerne les choses communes ; après quoi, il sera possible de déceler la signification de la naturalité du génome mise en rapport avec la notion de communauté.

\section{Les choses communes et l'ambiguïté de la référence à la nature}

7 Si le recours fait par l'Unesco à la notion de patrimoine commun de l'humanité constitue une référence claire à une théorie du patrimoine à présent éprouvée, quoique récente, elle doit aussi être mise en regard d'un concept de droit romain, celui de choses communes. D'inspiration et d'origine différentes, ces notions - patrimoine commun de l'humanité et choses communes - visent toutes deux à faire échapper l'entité sur laquelle elles s'appliquent à l'appropriation privée ; elles ne s'en distinguent pas moins nettement l'une de l'autre en ce que le patrimoine de l'humanité ne se situe pas franchement en dehors du cadre de la propriété, quand bien même ce serait un sujet de droits encore en voie de constitution - l'humanité - qui serait ainsi érigé en propriétaire.

8 La notion de "patrimoine commun de l'humanité », formulée en tant que telle pour la première fois en 1967 par A. Pardo en prévision de la Conférence de l'Organisation des Nations Unies sur le droit de la mer, vise à assurer une gestion pacifiste, utilitaire mais prévoyante, de certains biens considérés comme essentiels pour l'humanité actuelle et à venir (la lune, l'Antarctique, les ressources végétales et animales, le patrimoine architectural classé par l'Unesco, etc.) ${ }^{3}$. C'est cette notion, très critiquée depuis, soit pour 
son hypocrisie (elle n'empêcherait en aucun cas l'exploitation de l'entité considérée), soit pour son caractère purement incantatoire, que l'Unesco a reprise pour qualifier le génome humain ${ }^{4}$.

9 Il est vrai qu'elle l'a fait en insistant sur le caractère "symbolique » de la qualification, ce qui n'était pas le cas dans les premiers projets de la Déclaration. C'est ainsi que, dans la version de l'automne 1995, le génome était conçu comme «une composante fondamentale du patrimoine commun de l'humanité » et que, dans l'un des rapports précédant ce projet, on avait précisé que «le concept de "patrimoine commun de l'humanité" s'étend pour la première fois à la spécificité même de l'humanité, en même temps qu'à ce qui est au plus intime des êtres humains, à savoir le génome de chacun d'eux ${ }^{5}$ ». On allait même jusqu'à considérer l'humanité non pas d'un point de vue philosophique mais comme une notion de portée juridique, l'humanité étant érigée en sujet de droit international, avec des droits et des responsabilités vis-à-vis d'elle-même et des générations à venir ${ }^{6}$. Entre-temps, on s'est aperçu des difficultés liées à la reconnaissance, au bénéfice de l'humanité, de la personnalité juridique, avec son corollaire - la titularité d'un patrimoine -, et l'on a pris acte des résistances politiques que suscite le concept. La qualification du génome comme patrimoine commun n'est donc qu'un symbole, qui plus est de faible envergure.

Symbolique ou juridique, cette caractérisation n'en traduit pas moins une idée sousjacente à l'ensemble de la réglementation sur le génome et qui mérite qu'on s'y attarde du point de vue ici adopté, celui de la construction de l'objet en droit. En effet, le but évident de la qualification est de permettre diverses activités portant sur le génome (recherches fondamentales et appliquées), tout en postulant de manière générale l'inappropriabilité du génome. Or celle-ci est implicitement déduite, dans le processus de qualification ainsi envisagé, d'une certaine «nature » du génome, à savoir le fait qu'il appartienne à l'espèce entière. À cet égard, du reste, la qualification de "patrimoine commun de l'humanité » peut être concurrencée, sur son propre terrain, par celle de choses communes.

11 En effet, beaucoup plus traditionnelle et connue des juristes est la qualification de choses communes issue du droit romain où elle était utilisée pour désigner les choses qui font l'objet d'un usage commun à tous les hommes, parce qu'elles échappent à toute appropriation de type privé. Ainsi en allait-il de l'air, de l'eau courante, de la mer et de l'étendue des grèves recouvertes par le plus haut flot d'hiver. Inappropriables, les choses communes (res communes) n'en faisaient pas moins l'objet d'un droit d'usage assez largement entendu pour que les hommes pussent en maîtriser, fût-ce provisoirement, des parcelles: on peut pêcher en mer, construire des cabanes sur le rivage. La notion de choses communes a été reprise par l'article 714 du Code civil qui dispose : «Il est des choses qui n'appartiennent à personne et dont l'usage est commun à tous. Des lois de police règlent la manière d'en jouir. "

12 Le génome fournit alors l'occasion de se demander quel peut être l'usage contemporain d'une telle notion, dès lors que le constat à partir duquel elle s'était développée en droit romain, à savoir le caractère inappropriable, par nature, de certaines choses, ne peut plus être fait dans les mêmes termes qu'il y a deux mille ans. En effet, aujourd'hui, si le constat de l'inappropriabilité de certaines choses n'est pas directement remis en question, en revanche le fondement de la règle offre matière à discussions d'autant plus décisives qu'elles aboutissent, en fin de compte, à conférer à l'inappropriabilité un spectre plus ou moins large. 

toute appropriation pour des raisons liées à leur nature physique (l'abondance de la mer, l'ubiquité de l'air), ce qui n'en fait pas des biens, au sens juridique du terme, c'est-à-dire des choses qu'il est utile et possible de s'approprier ${ }^{7}$. Notons toutefois que cette présentation elle-même doit être nuancée, des transformations de statut (de chose à bien ou vice versa) pouvant avoir lieu, et la situation de fait à l'origine de la qualification de choses communes (l'abondance par exemple) pouvant se trouver modifiée de façon contingente.

D'après une seconde opinion, l'article 714 du Code civil serait plus normatif que descriptif et la qualification de choses communes découlerait clairement d'une réflexion en termes jusnaturalistes ${ }^{8}$. En ce sens, les choses sont communes en raison de leur nature même qui ne se réduit pas à une détermination de leurs caractéristiques physiques, leur abondance par exemple. On voit alors que selon cette acception, la nature dont il est question englobe un jugement de valeur : une chose n'est plus commune par essence, elle doit l'être par nature (juridique). En effet, qu'une chose commune abondante vienne à se raréfier, c'est alors que le rejet de toute appropriation privative s'avérerait indispensable et qu'il faudrait instituer la chose en question comme chose commune. Au contraire, à s'en tenir à la première acception, la chose initialement commune car abondante aurait, dans ce cas de figure, changé de statut puisqu'elle serait devenue rare et donc susceptible d'appropriation privée. Le second raisonnement part, lui, de la présomption - certes réfragable -, selon laquelle ce qui est a priori destiné à tous doit le demeurer jusqu'à ce qu'il soit démontré de façon certaine que sa répartition privative serait plus avantageuse pour l'humanité entière ${ }^{9}$. Tant que joue la présomption, la chose doit demeurer commune (c'est sa nature juridique), quand bien même sa nature physique se serait modifiée.

En réalité l'on voit que ces deux opinions relatives au fondement de l'inappropriabilité des choses communes combinent, de façon certes différente, le factuel et le normatif, la «nature » physique et la reconstruction de celle-ci par le droit en fonction de diverses considérations, notamment l'utilité. Leur coexistence a par conséquent le mérite de mettre au jour, s'agissant de la catégorie des choses communes, dont le caractère dérogatoire est marqué, le savant mélange de référence à une certaine « naturalité » et de prescription juridique qui caractérise le discours juridique sur des entités ayant conservé un lien avec la nature. Il importe dès lors d'en venir à cette "naturalité » juridique, appliquée au génome et déduite d'une certaine communauté.

\section{« Naturalité » et communauté du génome}

Il apparaît que selon un tour de passe-passe, le caractère commun en droit du génome est, explicitement ou implicitement, déduit de son caractère commun "par nature", raisonnement contestable qui sera mis à l'épreuve de la notion juridique de «nature des choses ».

Lorsque l'Unesco qualifie, d'un point de vue symbolique, le génome de patrimoine commun de l'humanité, c'est, on l'a vu, en référence à un impératif général d'inappropriabilité, exalté afin de contrebalancer les dangers liés à la disponibilité du génome, corollaire inévitable des pratiques qui le prennent pour objet. Or, quel peut bien être le fondement d'une telle inappropriabilité ? Il semble qu'on ait ici affaire à un raisonnement «naturalisant» des plus sommaires, et en même temps imparable, consistant à déduire le caractère commun (en droit) du génome de son caractère commun 
à toute l'espèce, c'est-à-dire d'un point de vue biologique. C'est ainsi par une sorte de truisme que l'on parvient à énoncer que le matériel juridique est chose commune dans la mesure où il concerne l'ensemble de l'espèce ${ }^{10}$. On constate alors aisément que le caractère commun par nature de l'entité considérée (ici les séquences génétiques) est à la fois le fondement et la limite de la qualification juridique de choses communes : dans une telle perspective, ne seraient offertes à l'usage de tous les hommes que les séquences nécessaires à la définition du caractère humain de l'individu. On mesure sans peine les difficultés pratiques et théoriques que suscite une telle assertion qui non seulement rabat trop vite la norme sur le fait, la règle juridique sur le constat biologique, mais surtout suppose de prendre parti sur l'humanité de l'homme qu'il serait tout de même trop simple d'appréhender uniquement au moyen du concept d'espèce.

Le génome illustre donc bien l'aporie à laquelle conduit le raisonnement tautologique selon lequel une chose est commune en droit (stricto sensu ou en tant qu'appartenant au patrimoine commun de l'humanité) parce qu'elle est commune en fait. Une telle explication écrase la distance entre le fait et la norme qui caractérise toujours le jugement de droit, quand bien même s'agissant des entités naturelles, cet écart peut parfois être infime. Un bref détour par la notion de « nature des choses » permettra peutêtre de comprendre en quoi consiste ce décalage.

Même si, en ce qui concerne le génome humain, une qualification de type commun servant à poser l'inappropriabilité de ce dernier peut se justifier, ce n'est pas, semble-t-il, en plaquant ce caractère commun sur une nature qui serait elle-même commune. En effet, un tel raisonnement fait l'impasse du rôle joué par la référence à la nature des choses dans le processus juridique de la qualification. On restreindra le champ de la discussion à quelques indications sur la fonction de la nature des choses dans l'argumentation juridique, sans l'étendre au cadre théorique plus général dans lequel s'inscrit le débat sur la nature des choses, à savoir l'histoire de la place et de la signification du droit naturel dans les divers systèmes juridiques existants ou concevables ${ }^{11}$.

Il suffira, pour les besoins de la démonstration, de constater que la "nature des choses ", en droit comme en tout domaine dans lequel cette référence est appelée à intervenir, est l'expression de l'idée d'un «donné primordial, extérieur à l'esprit, qui lui oppose une résistance irréductible, mais aussi fournit à ses constructions un point d'appui inébranlable ${ }^{12}$ »; plus simplement, elle manifeste « la reconnaissance par le juriste d'un agencement de faits ou de circonstances qui une fois reconnu s'impose à lui soit dans l'ordre de la réalité physique qu'il imagine, soit dans l'ordre rationnel auquel il souscrit ${ }^{13}$ ». Le juriste est, en effet, souvent amené à s'interroger sur la nature de tel phénomène ou telle institution. Il a besoin de la qualifier pour trouver le régime qui lui est applicable. $\mathrm{Si}$ l'entité considérée est encore inconnue de lui, il va être conduit à en rechercher l'essence, ce qu'elle est, afin de la faire coïncider avec telle ou telle catégorie juridique. Ainsi, dans le but de réglementer telle pratique contractuelle nouvelle, on la décompose selon les diverses opérations qu'elle réalise, pour faire entrer, autant que possible, chacune de celles-ci dans un moule juridique connu. Du coup, chacun des types de contrat utilisés perdra de sa nature originaire et subira des déformations par rapport à son régime ordinaire.

21 La controverse contemporaine à propos du couple homosexuel fournit un autre exemple, moins neutre eu égard à la référence à la "nature », des contraintes du processus de la qualification juridique. Envisagé comme l'union de deux personnes qui vivent comme des 
époux sans être mariées, le concubinage est appréhendé par référence à l'institution connue (le mariage), qu'il s'agisse de lui faire produire moins d'effets que ce dernier, ou d'exclure du concubinage ce qui ne s'apparente pas au mariage (l'union de deux personnes de même sexe). Toutefois, les difficultés suscitées aujourd'hui par les revendications tendant à la reconnaissance du concubinage homosexuel montrent bien le passage d'un raisonnement essentialiste à un raisonnement en termes de "nature des choses ", ainsi que l'ambivalence de cette dernière. En effet, dire que le mariage est l'union de deux personnes de sexe différent ${ }^{14}$ semble relever de prime abord de la description de l'essence d'une institution - de ce qu'elle est - mais de là on glisse très vite à la considération d'une « nature ». Dans le vocabulaire même, l'union libre, et la filiation qui en découle, sont considérées comme naturelles, par opposition à une situation légale (le mariage et la filiation légitime). Est naturelle ici l'union sexuelle qui n'est pas sanctionnée par la loi. Ainsi, pour trouver ce qu'est le concubinage, on commence par se référer à une nature des choses entendue comme un donné primordial et antérieur au droit (une sorte d'essence figée). Toutefois, celui-ci recouvre très rapidement son empire, et c'est là qu'entre en scène, semble-t-il, la nature des choses juridiquement métamorphosée. Si dans un état de nature indéterminé et confus, hommes et femmes procréaient sans le sceau de la loi, il parait de la nature (juridique) des choses, mixte de l'observation du monde extérieur et de la nature de l'homme, que seuls l'homme et la femme puissent être reconnus comme formant une union légitime. La nature des choses fournit ainsi une référence très commode au juriste pour justifier telle ou telle solution. Cette référence apparait tout à la fois comme le socle de la qualification et comme une limite possible au champ de l'intervention humaine, comme un point de départ (descriptif) et un point d'arrivée (normatif). En ce sens elle peut paraître tautologique mais il faut bien constater qu'« un juriste ne se sent ordinairement satisfait d'une situation que quand il a réussi à se convaincre qu'elle répondait à une nature des choses ${ }^{15}$ ", car cela présente à ses yeux le double avantage de l'équité et de la prévisibilité. Toutefois, il ne faudrait pas en conclure que la référence à une nature des choses implique pour le juriste une démission. Si, d'une certaine façon, invoquer une telle référence, c'est se dédouaner sur la morale commune d'un certain parti pris, en revanche la désignation même de cette nature en appelle à une observation des réalités extérieures au sein desquelles le juriste doit effectuer un tri, « un travail de l'intelligence qui dégage les structures et démêle ce que leur intelligibilité appelle ${ }^{16} \%$. Par conséquent, qu'on l'approuve ou qu'on la récuse, et ce pour des raisons de méthode ou de fond tenant essentiellement à la conception des rapports que doit entretenir le droit avec d'autres systèmes normatifs, il ne faut pas perdre de vue le fait que la "nature» du juriste est construite et qu'elle ne résulte pas de la simple observation d'une insaisissable réalité (la nature des choses) à laquelle, finalement, on peut faire dire tout et son contraire. Il est insuffisant de s'appuyer sur la seule «nature des choses » pour décider que l'unique union possible est celle de l'homme et de la femme; de même, la mer n'est pas une chose commune simplement parce qu'elle est abondante, cette dernière caractéristique étant relative et contingente. Il ne s'agit pas de prétendre que le droit doit bannir toute référence à la nature mais que pour être efficace juridiquement, cette référence doit passer par un remodelage qui signe, tout simplement, le passage de l'être au devoir-être, de la nature des choses à la nature juridique.

À ce titre, les qualifications naturalisantes du génome, au-delà de leur incontestable part d'exactitude en ce qu'elles reflètent bien un aspect de la réalité - la communauté du 
génome -, sont insuffisantes eu égard à la complexité de l'objet étudié dont elles ne parviennent pas à épuiser toutes les dimensions.

\section{Les insuffisances des qualifications « naturalisantes » du génome}

L'idée d'une "naturalité » première du génome, qui est au fondement du principe, incontestablement opportun, de son inappropriabilité, est malheureusement insuffisante tant en extension qu'en compréhension.

\section{La qualification conçue en extension}

En se fondant de façon plus ou moins explicite sur le fait que les membres de l'espèce humaine ont en partage un certain nombre de gènes, la qualification du génome comme " patrimoine commun de l'humanité » vise essentiellement le génome collectif. L'espèce, qui a fait son apparition pour la première fois dans le Code civil en 1994 par le biais de la nécessaire protection de son intégrité ${ }^{17}$, apparaît ainsi comme la version biologique juridicisée de l'humanité. Assurément, il est indispensable de protéger le génome de l'espèce contre certaines pratiques dont on maitrise encore mal les effets, comme la thérapie génique germinale qui transmet à la descendance de l'individu la modification subie par ce dernier; à ce titre, la qualification retenue par l'Unesco peut présenter des vertus. Toutefois, en l'état actuel, elle laisse dans l'ombre toute une série de problèmes, à savoir ceux que pose la mise à disposition du génome individuel ${ }^{18}$, plus particulièrement par le biais de pratiques qui, en réalité, se situent à la charnière de l'individuel et du collectif. De cette frontière floue, l'embryon constitue un bon exemple. On n'évoquera ici qu'une seule des techniques qui le prennent pour objet, à savoir le diagnostic préimplantatoire, afin de mettre en lumière les difficultés de catégorisation qu'il suscite.

Rappelons que le diagnostic préimplantatoire consiste, à la suite de la production d'embryons in vitro en vue de la fécondation d'une femme stérile ou de la procréation au sein d'un couple présentant de hauts risques de transmettre à sa descendance une maladie génétique, à effectuer un tri afin de n'implanter dans le corps de la femme que les embryons sains. Objet d'une vive polémique finalement tranchée par le législateur sur le mode de l'autorisation à titre exceptionnel ${ }^{19}$, le diagnostic pré-implantatoire ne concerne, à première vue, que quelques embryons singuliers, autrement dit des patrimoines génétiques individuels. On est loin, par conséquent, du patrimoine génétique de l'espèce conçu comme devant être commun à l'humanité et, partant, inappropriable. Tel est, du reste, l'argument utilisé par ceux qui, a priori, ne s'opposent pas à tout type d'intervention sur le génome, et notamment au diagnostic préimplantatoire: il serait fâcheux de sacraliser le génome, de l'ériger en patrimoine intangible. Pourtant, sans considérer comme inévitables les effets de chaîne d'une telle pratique (aujourd'hui une maladie grave, demain un handicap léger) et sans imaginer que la sélection des embryons puisse porter atteinte de façon décisive à l'intégrité de l'espèce humaine qui, de toute façon, est en constante évolution, on ne saurait occulter le fait que derrière les apparences, ce n'est pas seulement de tel embryon qu'il s'agit mais d'un ensemble de cellules à peine indifférenciées qui est bien de la mouvance de tel couple et, à ce titre, individualisable mais qui, avant implantation, reste dans une relative indétermination. En effet, au stade de l'éprouvette, l'embryon est voué à un sort incertain qui soit 
l'individualisera en le rattachant à un couple, qu'il s'agisse du couple d'origine ou d'un couple d'adoption $^{20}$, soit le fera basculer dans une maitrise de type collectif, parce qu'il sera détruit pour diverses raisons, après avoir fait, le cas échéant, l'objet d'une étude scientifique $^{21}$. Écartelé entre l'individu qu'il n'est qu'en puissance et l'humanité qu'il représente assurément d'un point de vue biologique mais à laquelle ne peut être exactement assimilée l'humanité juridique, l'embryon n'est donc pas vraiment concerné par la qualification du génome comme patrimoine commun de l'humanité, qui demeure trop générale pour s'appliquer de manière pertinente à une entité dont le traitement collectif ne saurait faire disparaitre l'irréductible individualité.

Ainsi, la qualification retenue par l'Unesco, en ce qu'elle a vocation à s'appliquer au génome de l'espèce, manque une partie de ses cibles potentielles, à savoir les techniques qui combinent sans pouvoir les dissocier l'individuel et le collectif. Insuffisante en extension, elle l'est aussi en compréhension, c'est-à-dire si l'on s'en tient au seul champ qu'elle concerne explicitement.

\section{La qualification conçue en compréhension}

On a constaté que la qualification du génome comme patrimoine commun de l'humanité était avant tout la simple traduction juridique du constat selon lequel d'un point de vue biologique, les membres d'une même espèce ont en commun certains gènes. En ce sens, cette qualification est "naturalisante» au sens premier du terme: la communauté juridique qu'elle vise à instituer par le biais de l'inappropriabilité serait purement et simplement justifiée par le caractère commun dans la nature de l'entité considérée. Or une telle naturalité du génome présente au moins deux inconvénients : il est des interdits qu'elle ne saurait fonder efficacement; elle postule une conception du génome que dément un grand nombre de pratiques.

En premier lieu, si le patrimoine commun de l'humanité a pour finalité, comme toutes les qualifications, de conduire à l'établissement d'un régime et si, comme on peut le supposer, ce dernier doit associer de façon harmonieuse des libertés (celle de la recherche notamment) et des interdits (celui de la discrimination principalement), alors force est de constater que la qualification retenue sera d'une efficacité variable. On ne donnera ici que deux exemples opposés.

La qualification est incontestablement utile - et tel est du reste l'un de ses buts avoués pour ériger des obstacles aux revendications des chercheurs qui prétendent breveter des gènes dont ils ignorent encore la fonction. En effet, la volonté d'appropriation par le mécanisme du brevet est ici barrée par le principe d'inappropriabilité, bien adapté au cas de figure, puisque de telles demandes portent sur rien de moins que sur une entité brute, non encore décodée par la science. La qualification du génome comme patrimoine commun de l'humanité peut alors venir au secours de l'idée selon laquelle seules les inventions sont brevetables, à l'exclusion des simples découvertes ${ }^{22}$.

En revanche, l'efficacité de la qualification de patrimoine commun semble plus douteuse quand on la confronte à une pratique comme la xénogreffe qui cherche, de manière encore expérimentale, à dépasser, dans le domaine des transplantations, la barrière de l'espèce. Dans l'hypothèse où l'on jugerait utile de prohiber une telle pratique, l'on voit combien le raisonnement naturalisant serait faible. Si le patrimoine génétique doit être déclaré commun à l'humanité parce qu'il est commun à l'ensemble de l'espèce humaine et qu'en cela il est porteur de valeurs universelles, alors le fait que nous partageons $90 \%$ de 
nos gènes avec les singes pourrait nous inciter à faire produire des effets juridiques à notre communauté avec le règne animal, surtout si cette communauté biologique est utile (par exemple, dans le cas des greffes, à des fins thérapeutiques). Qui ne voit que la qualification naturalisante est réductrice et qu'elle conduit au résultat opposé à celui qu'elle visait?

31 Prise en un sens littéral, la qualification retenue par l'Unesco présente donc un risque non négligeable d'inefficacité, conséquence de son évidence qui n'est qu'apparente, comme si le fait d'appartenir à une même communauté - l'humanité - allait de soi et ne méritait aucune explicitation, et ce alors qu'une technique comme la xénogreffe suffit à prouver le contraire.

32 En second lieu, cette qualification comporte un défaut moins fondamental mais plus immédiatement gênant, son manque de réalisme juridique. En effet, on ne saurait se satisfaire de la représentation véhiculée par cette qualification, à savoir celle d'une communauté de gènes qui unirait, au même titre que le patrimoine naturel ou culturel ${ }^{23}$, tous les membres du genre humain. Une telle conception faisant du génome une entité normalement accessible à nos sens et à notre connaissance méconnaît sa profonde artificialité. Or on ne voit guère ce qu'il y a de commun entre la lune, par exemple, et les résultats obtenus par le séquençage du génome. Une véritable différence de nature sépare les choses aisément intelligibles, qu'elles soient matérielles ou non, et ce pur produit d'une science très sophistiquée qu'est le génome. La qualification de patrimoine commun est sans doute séduisante par le rapprochement qu'elle tente d'opérer entre des entités de nature différente mais servant toutes, à un titre ou un autre, au bien-être de l'humanité; elle n'en est pas moins fallacieuse, parce qu'elle gomme le caractère essentiellement artefactuel du génome.

Par conséquent, elle ne permet pas de penser les nouveaux rapports entre nature et artifice auxquels aboutit la mise à disposition du génome humain, et que les procréations médicalement assistées mettent particulièrement bien en relief. Certes, comme on l'a déjà souligné, la qualification du génome humain comme patrimoine commun de l'humanité laisse en partie dans l'ombre le patrimoine génétique individuel, directement concerné par les nouvelles techniques de reproduction destinées à pallier la stérilité d'un couple ou à éviter la transmission d'une maladie génétique grave ${ }^{24}$. Malgré tout, on peut ici procéder à une transposition du raisonnement mené à propos du manque de réalisme de la représentation sous-jacente à la qualification de type commun. En effet, à première vue, les techniques en question tentent d'imiter les processus naturels et contribuent ainsi à la perpétuation de ce patrimoine génétique que nous avons tous en partage. Toutefois, au-delà de ce simple constat, on doit souligner le fait que les nouveaux moyens mis en œuvre pour que l'espèce humaine se reproduise bouleversent de fond en comble la conception du génome - avatar moderne de l'hérédité - véhiculée par chacun des modes de transmission de la vie. Pourtant, tout se passe comme si, du point de vue de leur réglementation juridique et de leur acceptation par le corps social, les techniques très performantes dont nous disposons ne parvenaient pas à s'affranchir de la référence à la nature, de même que la qualification du génome comme patrimoine commun de l'humanité prend appui sur une certaine communauté biologique naturelle. Or le refus d'assumer franchement le caractère artificiel de la transmission dans le cadre d'une procréation assistée est à l'origine de règles très contestables pour des raisons tant juridiques qu'éthiques, notamment celle de l'anonymat du donneur de gamètes, dans l'hypothèse d'une insémination artificielle avec tiers donneur. L'anonymat sert ici à 
masquer l'inacceptable, à savoir l'écart à la nature que maintient une technique consistant précisément à remédier aux insuffisances de la nature. On peut dès lors se demander si, dans ce dispositif, la nature continue de constituer une référence et, si oui, avec quelle signification ${ }^{25}$. De même, l'idée naturalisante qui se trouve au fondement de la qualification du génome comme patrimoine commun renvoie à un référent - une communauté biologique - trop flou pour fonder des solutions juridiques efficaces.

Ainsi, faute d'une définition renouvelée des notions d'humanité et de nature, la qualification de type commun est, en l'état, insuffisante : elle postule trop vite un accord sur la notion d'humanité que l'apparition de nouvelles techniques rend pourtant ambiguë; elle se fonde sur l'idée, juste mais vague, d'une commune nature, au lieu d'assumer franchement le caractère artefactuel du génome et de réglementer ce dernier en tant que tel. En bref, elle présente deux défauts opposés: elle n'anticipe pas suffisamment sur les problèmes que ne manqueront pas de poser certaines techniques; elle ne prend pas acte des modifications irréversibles que la biologie moderne a imposées à notre vision des rapports entre la nature et l'artifice.

En conclusion de cette présentation critique de la qualification du génome comme patrimoine commun de l'humanité, il est juste de retoucher le tableau dans le sens de la nuance.

Tout d'abord, répétons que cette qualification n'a, selon les auteurs de la Déclaration, qu'une valeur symbolique. Toutefois, cette mesure de prudence est quelque peu gênante car elle revient à enlever d'une main ce qui a été donné de l'autre (un régime juridique), ce qui est pour le moins incohérent. En droit, il n'y a pas de qualification symbolique. Par conséquent, soit la Déclaration a une simple vocation morale et elle n'intéresse guère le juriste ; soit ce dernier la prend au sérieux, dans sa double dimension morale et juridique, auquel cas il doit l'investir d'un sens acceptable et qui conduise à des solutions efficaces.

Ensuite, la qualification retenue par la Déclaration reflète bien les problèmes suscités par l'appréhension d'objets récemment entrés dans l'orbite du droit, et notamment d'entités complexes comme le génome. Mieux, elle nous permet de constater, sur la très longue durée, une relative permanence dans les difficultés rencontrées pour qualifier les entités "naturelles ", c'est-à-dire celles qui préexistent au droit et à l'action technique de l'homme, comme les rivières, le corps ou, dans une moindre mesure, le génome. Ici, plus qu'ailleurs, le droit hésite à s'affranchir de toute référence aux caractéristiques de l'entité préexistante, et ses qualifications portent la marque d'une tension entre le souci d'adhérer aux faits et celui de les transmuer pour mettre en évidence l'autonomie du jugement de valeur. Que dans notre société le droit soit toujours pris dans cet étau est certes un signe des limites de notre acceptation sociale de la technique, comme si, d'un point de vue conceptuel, la nature n'était jamais entièrement recouverte par la technique qui l'a transformée. Toutefois, on peut plutôt penser que, dans le domaine des objets naturels métamorphosés en artefacts, ce sont les référents de la qualification juridique qui devraient être reformulés. Plus précisément, même lorsque sont en jeu la génération et la barrière de l'espèce, autrement dit les plus anciens signes d'un immuable ordre des choses, la " nature des choses » chère aux juristes, si elle doit encore servir de référent, devra être conçue comme un réel tout ensemble naturel et technique, comme un existant dont le droit a besoin. En effet, bien que l'inventivité de ce dernier soit sans doute sans 
limite, à l'instar de celle de toute technique, on ne voit pas qu'elle puisse s'exercer à partir de rien. Assumant l'artificialité de son objet, la qualification du génome pourrait contribuer à l'émergence d'une « nature des choses » comme référent de base susceptible d'aboutir, après une reconstruction juridique, à des solutions efficaces, et non comme un refuge pour juristes à juste titre désemparés.

\section{NOTES}

1. Article premier de la Déclaration universelle sur le génome humain et les droits de l'homme, adoptée par l'Unesco le 11 novembre 1997, lors de la $29^{\mathrm{e}}$ session de la Conférence générale, Paris, Dictionnaire permanent de bioéthique et de biotechnologies, bull. 55 (6 décembre 1997), p. 8713.

2. Cf. D. Bourg, L'homme artifice, Paris, Gallimard, 1996, p. 20 sq., expliquant à quel point la biologie moléculaire a profondément modifié la catégorie d'artefacts constituée par les objets naturels que nous modifions (l'autre catégorie comprenant les objets techniques que nous fabriquons).

3. Cf. A.-C. Kiss, "La notion de patrimoine commun de l'humanité ", in Recueil des cours de La Haye [1982, II], tome 175, 1983 ; M. Flory, «Le patrimoine commun de l'humanité dans le droit international de l'environnement », in Droit et environnement. Propos pluridisciplinaires sur un droit en construction, avant-propos de A. Sériaux, Aix-en-Provence, Presses universitaires d'AixMarseille, 1995, p. 39-50 ; J. Charpentier, «L'humanité : un patrimoine, mais pas de personnalité juridique », et P. Kahn, «Les patrimoines communs de l'humanité. Quelques réflexions ", in Les hommes et l'environnement. En hommage à Alexandre Kiss, Paris, Frison-Roche, 1998, p. 17-21 et p. 307-314.

4. Pour une critique, voir B. Edelman, «Entre personne humaine et matériau humain : le sujet de droit ", in B. Edelman et M.-A. Hermine, eds, L'homme, la nature et le droit, Paris, C. Bourgois, 1988, p. 136-141.

5. Rapport de la quatrième réunion de la commission juridique du CIB (Comité international de bioéthique), p. $3, \mathrm{n}^{\circ} 8$.

6. Ibid.

7. Cf. F. Zénati et T. Revet, Droit civil. Les biens [1988], Paris, Presses universitaires de France, 1998, p. $12, \mathrm{n}^{\circ} 27$.

8. Cf. A. Sériaux, «La notion de choses communes. Nouvelles considérations juridiques sur le verbe avoir ", in Droit et environnement. Propos pluridisciplinaires sur un droit en construction, op. cit., p. 39-50, en particulier p. 28.

9. Ibid., p. 29-30. Ainsi, entre l'air, l'eau courante ou la lumière, d'une part, et la terre, d'autre part, il y a cette différence que, au moins en l'état actuel des choses, l'usage et l'exploitation des premiers n'exigent pas, pour être efficaces, une possession privative, alors que la seconde nécessite au contraire, toujours dans les mêmes buts, une répartition entre les personnes.

10. Pour ce raisonnement appliqué en un sens restrictif, c'est-à-dire pour réduire le champ de l'inappropriabilité, voir N.-J. Mazen, «Problèmes juridiques liés aux applications médicoindustrielles de la carte génétique humaine », Revue de la Recherche juridique, Droit prospectif, 1991-2, p. 370.

11. Sur les rapports (ou leur absence) entre droit naturel et nature des choses, voir H. Batiffol, $\mathrm{La}$ philosophie du droit [1960], Paris, Presses universitaires de France, 1981 [6éd.], p. 55-78; Droit et 
nature des choses. Travaux du colloque de philosophie du droit comparé (Toulouse, 16-21 septembre 1964), Paris, Dalloz, 1965 ; P. Foriers, «La motivation par référence à la nature des choses ", in La pensée juridique de Paul Foriers, Bruxelles, Bruylant, 1982, vol. II, p. 795-812.

12. L. Husson, "Approches et aspects divers de la nature des choses ", in Droit et nature des choses, op. cit., p. 37-38.

13. P. Foriers, op. cit., p. 812.

14. Cf. en dernier lieu, civ $3^{\text {e }}, 17$ décembre 1997, Dalloz 1998, p. 111, conclusion J.-F. Weber, note J.-L. Aubert.

15. H. Batiffol, op. cit., p. 74.

16. Ibid., p. 75.

17. Cf. l'article 16-4 al. 1 du Code civil: «Nul ne peut porter atteinte à l'intégrité de l'espèce humaine ».

18. Notons que les auteurs de la Déclaration ont bien évidemment pensé à la protection du patrimoine génétique individuel, par exemple dans l'article 2 qui énonce le principe du respect de la dignité et des droits de chaque individu, quelles que soient ses caractéristiques génétiques, et qui s'oppose au réductionnisme génétique. Par ailleurs, on pourra se reporter au document intitulé «Consultation internationale sur l'esquisse de Déclaration de l'Unesco sur le génome humain » du 5 avril 1996 (CIP/BIO/96/COMJUR. 6/4), p. 2-3, sur le point de savoir si la notion de patrimoine commun de l'humanité serait susceptible de limiter le droit de l'individu de disposer librement de son patrimoine génétique, notamment de le transmettre à sa descendance, par exemple dans l'hypothèse où un État mènerait une politique autoritaire en matière de santé publique.

19. Cf. l'article L. 162-17 du Code de la santé publique.

20. Cf. les articles L. 152-3 à L. 152-5 du Code de la santé publique.

21. Cf. l'article L. 152-8 al. 3 du Code de la santé publique.

22. Pour une manifestation typique des difficultés suscitées par la matière, cf. la rédaction embarrassée du nouvel article L. 611-17 du Code de la propriété intellectuelle disposant que « le corps humain, ses éléments et ses produits ainsi que la connaissance de la structure totale ou partielle d'un gène humain ne peuvent, en tant que tels, faire l'objet de brevets ».

23. Pour un exemple d'assimilation contestable entre les divers patrimoines de l'humanité, cf. Conférence générale de l'Unesco, $28^{\mathrm{e}}$ session, Résolution 28 C/O.12 du 13 novembre 1995, sur la "Stratégie à moyen terme pour 1996-2001», affirmant «l'urgence de renforcer la solidarité morale de l'humanité pour assurer la sauvegarde du patrimoine qui lui est commun, naturel et culturel, matériel et immatériel, intellectuel et génétique » (paragraphe 6).

24. Voir l'article L. 152-2 al. 2 du Code de la santé publique.

25. Pour une analyse des références explicites et implicites de la réglementation des procréations médicalement assistées, voir M. Iacub, «La construction juridique de la nature dans la reproduction hors-nature : les fécondations artificielles dans les lois bioéthiques ", in F. Ronsin, H. Le Bras et E. Zucker-Rouvillois, eds, Démographie et politique, Dijon, Éditions universitaires, 1997, p. 161-173. 


\section{RÉSUMÉS}

Le génome humain, à la fois œuvre de la nature et production technique, illustre clairement les difficultés de la qualification juridique des objets, procédé qui, tout en décollant du réel, s'en défait avec peine lorsque ce socle est constitué par la nature au sens le plus commun du terme. La qualification «symbolique » récente qui en a été donnée par l'Unesco, celle de "patrimoine commun de l'humanité ", révèle bien, avec ses mérites et ses défauts, les méandres d'une construction juridique des entités naturelles qui permette d'éviter à la fois les pièges du naturalisme et ceux de l'artificialisme.

The human genome, both a natural product and a technical produce, clearly illustrates the difficulties of the juridical definition of objects. The process, which dissociates the objects from their reality, only gets rid of this reality with difficulty when it is constituted by nature in the most common sense of the term. The «symbolic » definition of the genome recently given by UNESCO - the " common patrimony of humanity " - clearly reveals the intricate patterns of a juridical construction of natural entities which permits avoiding the traps of naturalism as well as those of artificialism.

\section{AUTEUR}

\section{FLORENCE BELLIVIER}

Florence Bellivier (Université de Paris I) enseigne le droit civil. Elle a participé à divers ouvrages relatifs à l'histoire du droit de la famille (Naissance du Code civil, sous la direction de F. Ewald, Paris, Flammarion, 1989 ; La Famille, la Loi, l'Etat, sous la direction de I. Théry et C. Biet, Paris, Imprimerie nationale - Centre Georges-Pompidou, 1989). Elle vient de publier Le patrimoine génétique humain : étude juridique (Paris, L.G.D.J., 1999). 